\title{
Dissection of the Genetic Architecture of Rice Tillering using a Genome-wide Association Study
}

Su Jiang ${ }^{1,2 \dagger}$, Dan Wang ${ }^{1 \dagger}$, Shuangyong Yan ${ }^{3}$, Shiming Liu², Bin Liu ${ }^{4}$, Houxiang Kang ${ }^{2 *}$ and Guo-Liang Wang ${ }^{2,5^{*}}$ (D)

\begin{abstract}
Background: Rice tiller number (TN) is one of the most important components associated with rice grain yield. Around one hundred rice TN genes have been identified, but dissecting the genetic architecture of rice TN variations remains difficult because of its complex trait and control by both major genes and quantitative trait loci (QTLs).

Results: In this study, we used a subset of the rice diversity population II (S-RDP-II), genotyped with 700,000 single nucleotide polymorphisms (SNPs), to identify the loci associated with tiller number variations (LATNs) through a genome-wide association study (GWAS). The analysis revealed that 23 LATNs are significantly associated with TN variations. Among the 23 LATNs, eight are co-localized with previously cloned TN genes, and the remaining 15 LATNs are novel. DNA sequence analysis of the 15 novel LATNs led to the identification of five candidate genes using the accessions with extreme TN phenotypes. Genetic variations in two of the genes are mainly located in the promoter regions. GRT-PCR analysis showed that the expression levels of these two genes are also closely associated with TN variations.

Conclusions: We identified 15 novel LATNs that contribute significantly to the genetic variation of rice TN. Of these 15, the five identified TN-associated candidate genes will enhance our understanding of rice tillering and can be used as molecular markers for improving rice yield.
\end{abstract}

Keywords: Rice, Tiller number, Genome wide association study, Genetic architecture, Single nucleotide polymorphism, Linkage disequilibrium, Gene expression

\section{Background}

Rice (Oryzae sativa L.) is one of the main cereal crops that feeds more than half of world's population (Khush 2005). Tiller number (TN) and panicle morphology are two key factors associated with rice grain yields (Wang \& Li 2011). Rice TN is also used as a model system for studying the branching mechanisms in monocotyledonous plants (Li et al. 2003). Two developmental steps are used for regulating shoot branches: the first is the formation of axillary meristem, and the second is the growth of axillary buds (Yasuno et al. 2007). For a

\footnotetext{
*Correspondence: kanghouxiangcaas@163.com; wang.620@osu.edu

†'Su Jiang and Dan Wang contributed equally to this work.

${ }^{2}$ State Key Laboratory for Biology of Plant Diseases and Insect Pest, Institute

of Plant Protection, Chinese Academy of Agricultural Sciences, Beijing

100193, China

Full list of author information is available at the end of the article
}

particular variety of rice, its tillering capacity is largely determined by genetic factors and can be affected by environmental conditions, such as light, temperature, plant density, nutrients and water supply (Wang \& Li 2005).

Three important monoculm genes have been identified thus far in rice: $M O C 1, M O C 2$, and MOC3. MOC1 (Os06g40780) on chromosome 6 is a member of the GRAS (GAI, RGA and SCR) family of proteins that promotes axillary bud outgrowth. In the moc1 mutant, a 1.9$\mathrm{kb}$ retrotransposon inserted in $\mathrm{MOC} 1$ interrupts the gene and leads to a single main culm phenotype (Li et al. 2003). MOC2 (Os01g6466) on chromosome 1 encodes a fructose-1,6-bisphosphatase (FBP1), which is imperative for tiller bud outgrowth in rice. A Tos17 transposable element inserted into exon 4 of $M O C 2$ results in significantly reduced TN when compared to wild-type rice (Koumoto et al. 2013). MOC3 (Os04g56780)/TAB1/ 
OsWUS on chromosome 4 is requird for axillary buds formation (Lu 2015b). Some of the tillering dwarf genes that have been identified are also associated with effective TN by way of the strigolactone signaling pathway. For example, D3 (Os06g06050) on chromosome 6 encodes an F-box leucine-rich repeat (LRR) protein, which controls the axillary bud activity (Shinji et al. 2005). $d 27$ (Os11g0587000, IRGSP-1.0) on chromosome 11 encodes an iron-containing protein, which regulates rice tiller bud outgrowth (Lin et al. 2009). HTD2/D88/D14 (OsO3g1 0620) on chromosome 3 encodes esterase/lipase/thioesterase, which negatively regulates tiller bud outgrowth (Liu et al. 2009). D53 (Os11g01330) on chromosome 11 is a repressor of strigolactone signaling (Jiang et al. 2013). Genes in the plant hormone pathway are also involved in TN regulation, including the cytokinin oxidase/dehydrogenase gene OsCKX2 (Os01g10110) (Yeh et al. 2015), the caroteniod isomerase gene MIT3 (Os11g36440) (Liu et al. 2017) and the putative auxin efflux carrier OsPIN1 (Os02g50960) (Xu et al. 2005). Furthermore, the amino acid transporter OsAAP3 (Os06g36180) negatively regulates bud outgrowth and rice TNs (Lu et al. 2018), and heme activator protein gene OsHAP2E (Os03g29760) increases photosynthesis and TNs (Alam et al. 2015).

Rapid development of both next-generation genome sequencing and genotyping technologies supports construction of high-density SNP maps for a population that is both technically feasible and cost effective. The genome-wide association study (GWAS) method based on the high-density SNP maps and large diverse germplasm collections has become a new strategy for efficient and high-throughput gene identification in multiple species (Liu \& Yan 2019). The first GWAS on 14 agronomic traits identified three loci associated with the TN (Huang et al. 2010). Another GWAS led to the identification of a TN-associated $200 \mathrm{~kb}$ size locus that included 32 canidate genes ( $\mathrm{Lu}$ et al., 2015c). Dozens of loci associated with both TN variation (Wu et al. 2018) and tiller angle-variation (Dong et al. 2016) were recently identified using different natural rice populations. Because TN is a complex trait and the genetic basis of tillering is not completely understood, more research is needed to fully understand the genetic architecture of rice $\mathrm{TN}$ variations and identify more loci associated with tiller numbers (LATNs) in diverse germplasms.

In this study, we investigated the rice TN using a subset of the rice diversity population II (S-RDP-II) (McCouch et al. 2016). We identified 23 LATNs that were significantly associated with TN variations. Among those 23 loci, 12 cloned TN-related genes are colocalized within the 8 LATN regions, and the remaining 15 loci are novel. Further genetic sequence comparison and gene expression analyses enabled us to identfy 5 novel TN-associated genes in the 15 novel LATNs. Our study revealed that, besides genetic sequences, gene expression levels are also important for rice $\mathrm{TN}$ variations. The novel LATNs identified in this study could be useful for further dissection of the complex genetic architecture of rice $\mathrm{TN}$ variations and molecular breeding of high yielding rice cultivars.

\section{Methods}

\section{Plant materials}

The rice diversity population and the high diversity SNP maps (700,000 SNPs) are publicly available (McCouch et al. 2016; http://www.ricediversity.org/data/).

\section{Field experiments}

All rice seeds were soaked in clean water, at $37^{\circ} \mathrm{C}$ for $36 \mathrm{~h}$, replaced with clean water every $9 \mathrm{~h}$, then germinated for $16 \mathrm{~h}$. The seeds were sowed in Wuqing, Hebei province on May 1, 2018, and the 25-day-old seedlings were transplanted into the field. Transplanting density was $16 \mathrm{~cm} \times 20 \mathrm{~cm}$ with three rows and eight seedlings. A complete randomized block design was used with three replicates for each accession. The three plants in the middle were investigated for the $\mathrm{TN}$ at the later tillering stage. The mean $\mathrm{TN}$ from three replicates was used for the GWAS.

\section{The GWAS method of the S-RDP-II population}

The GWAS method was similar to that previously described in our study (Kang et al. 2016), except using a new version of the Tassel program (Tassel 5.0). Briefly, the mixed linear model (MLM) was selected in which the genetic marker-based kinship matrix $(\mathrm{K})$, generated by Tassel with the SNP dataset, was used jointly with population structure $(\mathrm{Q})$. We selected candidate LATNs using the following standard: a genomic region with $\leq 200 \mathrm{~kb}$ with at least two significant associated SNPs ( $p$ value threshold $\leq 1 \mathrm{E}-4$ ).

\section{Candidate gene sequencing and sequence analysis}

The sequences of the five candidate genes were downloaded from the Rice Genome Annotation Project website (http://rice.plantbiology.msu.edu/analyses_search_ blast.shtml). The full-length sequence of the candidate genes contains from $\sim 1500 \mathrm{bp}$ upstream of $5^{\prime} \mathrm{UTR}$ to 500 bp downstream of 3'UTR. The Gene specific primers were designed for cloning those genes in the accessions which have extreme high TN and extreme low TN. DNA sequencing of the cloned genes was finished by the TSINGKE Company in Beijing. Sequence alignment was performed with DNAMAN. All primers used in the study are shown in Additional file 1: Table S1. 
RNA extraction and candidate gene expression analysis Rice leaf samples were collected from rice cultivars in the paddy field. RNA and DNA extraction were performed with Trizol reagent (Invitrogen) and CTAB buffer, respectively. cDNA synthesis was performed according to the manufacturer's instructions for the cDNA synthesis kit (Vazyme). qRT-PCR was performed on ABI Prism 7500 PCR instrument with $10 \mu \mathrm{l} 2 \times$ SYBR Green Mix (GeneStar), $5 \mu \mathrm{l}$ cDNA, $0.5 \mu \mathrm{l}$ gene-specific primers and $4 \mu$ filter water. Rice ubiquitin gene was used as an internal control for normalization. The qRT-PCR primers are listed in Additional file 1: Table S1.

\section{Results}

\section{TN variations in the S-RDP-II population}

The rice TN phenotype is associated not only with genetic elements but also with environmental conditions, such as light and nutrition (Wang \& Li 2005). Temperate Japonica (TEJ) rice belongs to the northern ecotype in China, and subtropical indica (IND) rice belongs to the southern ecotype. To investigate whether the growth conditions in the two regions affect rice tillering, we selected 10 rice accessions from the previously studied rice diversity population 1 (RDP1). Among the 10 accessions, five had low TN and five had high TN based on their phenotypes in our Langfang greenhouses in Beijing. We grew these 10 accessions in the field of both North China (Beijing) and South China (Changsha). Results showed that the TN of all the cultivars were similar between North China (Additional file 2: Figure S1) and South China (Additional file 2: Figure S2), indicating that hereditary factors, rather than growth conditions, are most crucial for rice $\mathrm{TN}$ variations.

To dissect the genetic structure of rice tillering, we selected 350 accessions based on two principles from the RDP-II that contained about 1500 accessions. First, we made a phylogenetic tree using the whole RDP-II population and manually checked the branches of the tree to make sure no closely related accessions were selected. We then increased the seeds of the RDP-II population in the field with subtropical climate and selected those with normal growth and enough seeds. The population of the selected 350 accessions (S-RDP-II) contained six subpopulations, including IND, TEJ, tropical japonica (TRJ), aromatic (ARO), aus (AUS) and admixture (ADM) with 145, 31, 72, 42, 53 and 7 accessions, respectively (Fig. 1a). We calculated the TN of S-RDP-II at the mature stage (Fig. 1b). The average TN of the population is 19.0, with a minimum of 5 (accession: 121017) and maximum of 76 (accession: 117434) tillers at the mature stage (Additional file 1: Table S2). The distribution of the TN in the population was nearly a normal distribution (Fig. 1c). About $52.3 \%$ accessions (183 of 350 ) had $12-20$ tillers. The average TN among the different sub- populations was similar, i.e., $19.0( \pm 8.8 \mathrm{SD}$ [standard deviation]), 20.0 ( $\pm 6.3 \mathrm{SD}), 17.5( \pm 8.2 \mathrm{SD}), 19.2( \pm 8.8 \mathrm{SD})$, $20.5( \pm 11.6 \mathrm{SD})$ and $20.0( \pm$ 7.0SD) in IND, TEJ, TRJ, ARO, AUS and ADM, respectively (Fig. 1d). The relatively big SD value in each subpopulation indicated that the $\mathrm{TN}$ variation is large within the subpopulations.

\section{Identification of LATNs in the rice genome}

Using the TN phenotypes obtained from the field in this study (Additional file 1: Table S2) and the 700,000 SNP maps of the S-RDP-II, we performed the GWAS and identified 23 LATNs (Table 1). The 23 loci were distributed onto all of the rice chromosomes except for 2, 8 and 9 (Fig. 2). A simple additive model in the Statistical Analysis System (SAS) was used to determine whether the genotypes of the 23 LATNs were correlated with the TN phenotypes. The 15 LATNs with the most significantly associated SNPs from the 23 LATNs were included in the model. The correlation analysis showed that the 15 LATNs explained $73.4 \%$ of the TN variation in the S-RDP-II population. The 15 loci are LATN-1, 2, $3,6,8,9,10,11,13,15,17,18,21,22$ and 23 (Table 1). These results indicate that multiple loci control the complex genetic architecture of the TN variation in rice.

When compared with 90 previously cloned TN associated genes (Additional file 1: Table S3), we found that 12 cloned genes were co-localized with 8 LATNs including LATN-2, 7, 8, 9, 10, 15, 17 and 19 and the other 15 loci were novel loci. Detailed information of the known genes is listed in Table 1. It is most notable that MOC3 is co-localized with LATN-10. A previous study showed that the single tiller mutant moc3 is caused by one SNP mutation (Lu et al., 2015b); we subsequently investigated whether the association at LATN-10 was due to MOC3's sequence polymorphism or not. We selected 5 accessions with few TN and 6 accessions with more TN that contained the corresponding genotype at the locus. Sequencing and sequence alignment of the full length fragment (including promoter and coding region) of $M O C 3$ identified 11 allelic variations: 10 SNPs and one 12-bp InDel (Additional file 1: Table S4), however, none of those variations was the same as the functional mutation in moc3 reported previously (Lu et al., 2015b). All of these variations were associated with the TN phenotypes. It is interesting to mention that all of these variations were located in the putative promoter region, indicating that the variations may be associated with MOC3's expression rather than its gene coding sequences.

\section{Gene ontology analysis of the 15 novel LATNs}

We analyzed the $200 \mathrm{~kb}$ genomic sequences $(100 \mathrm{~kb}$ flanking the left and right side of the most significantly associated SNPs) of the 15 novel LATNs from the 

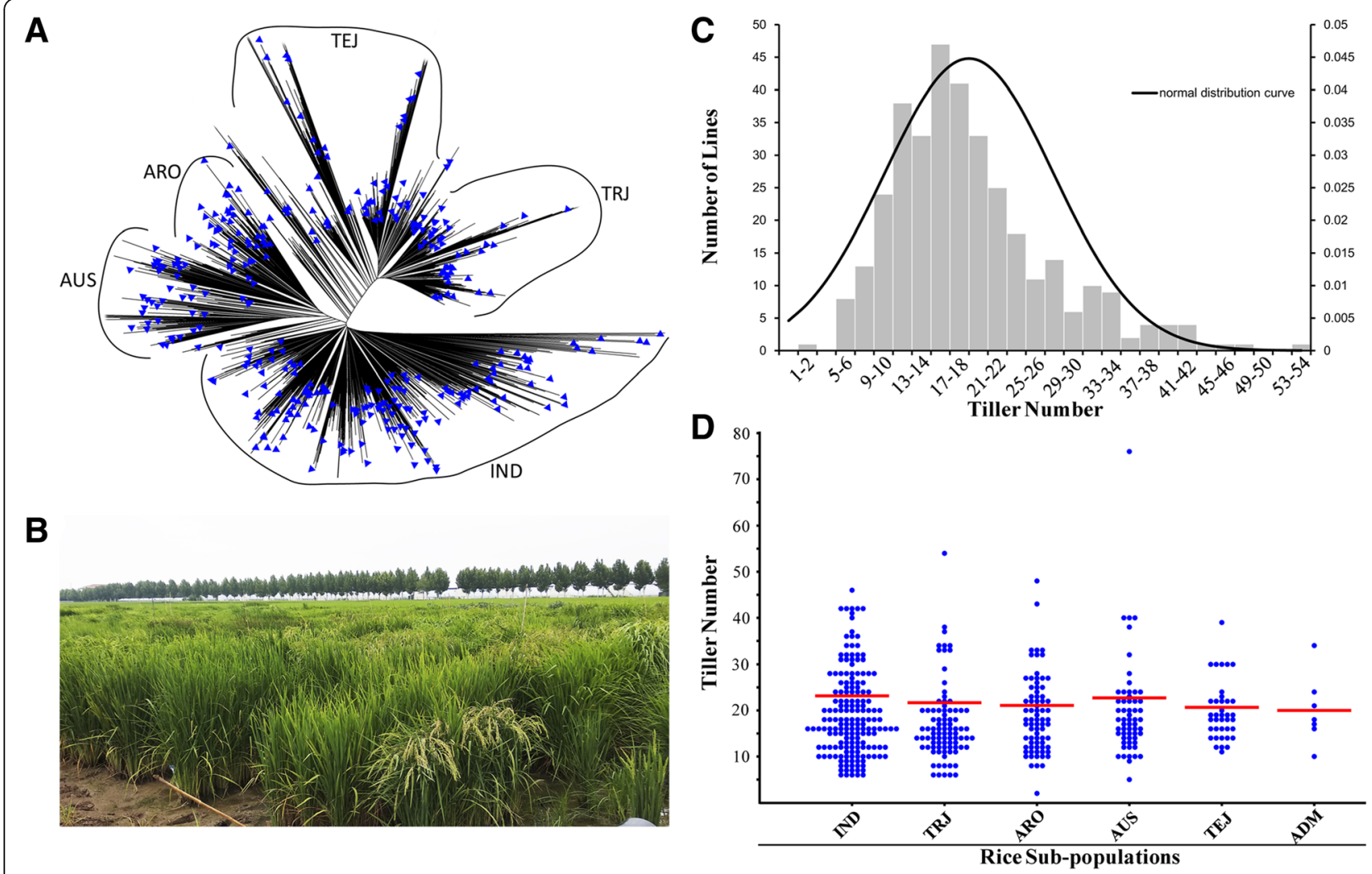

Fig. 1 The structure of the S-RDP-II population and its TN variation in the field. a Phylogenetic tree of the RDP-II population in which the 350 accessions (S-RDP-II) are indicated by blue triangles. Six subpopulations are IND, TEJ, TRJ, AUS, ARO and ADM. b Photo of S-RDP-II cultivars in the field when TN was counted. $\mathbf{c}$ The TN variation distribution in the S-RDP-Il population. X-axis represents TNs and Y-axis represents the number of accessions with the TN interval. $\mathbf{d}$ The TN variation in the subpopulations. Each dot represents a rice accession in the subpopulation. Red line represents the average value of TNs in the six subpopulations

reference genome of the japonica cultivar Nipponbare (NPB). These 15 loci encoded a total of 435 genes (Additional file 1: Table S5). Among these genes, 142, 98 and 36 genes were annotated as unknown proteins, retrotransposon and transposon proteins, respectively. The other 159 genes were annotated with putative functions using the gene annotation tool (http://www.ebi.ac.uk/ interpro/search/sequence-search). About 55\% (88 of 159) of the gene products belong to six classes: 39 enzyme proteins, 12 chloroplast and photosystem related proteins, 10 protein kinases, 10 NBS-type proteins, six $\mathrm{NADPH}$-dependent oxidoreductase, four transcription factors, four plant hormone related proteins and three cytochrome P450.

\section{Five novel genes associated with TN variations}

To further validate the association between the candidate genes and the TN phenotypes, we selected seven genes (Additional file 1: Table S6) either in the center of the peak value region of the associated SNPs or annotated as plant hormone-related genes from the 15 novel LATNs for sequencing and quantitative real time polymerase chain reaction (qRT-PCR) analysis. We designed the specific primers for each candidate gene (Additional file 1: Table S1) and amplified all of the genes, except Os05g14010 at LATN-11 and Os11g37840 at LATN-22, which could not be amplified by their specific primers in most of the tested rice accessions. The five genes were Os01g28690 at LATN-3, Os05g32120 at LATN-12, OsO7g28890 at LATN-14 and Os11g15130 and Os11g15210 at LATN-21. The genomic sequencing results confirmed that all five of the genes contained SNPs which were associated with the $\mathrm{TN}$ variation. qRT-PCR using the extreme low and high TN varieties and "two sample t-test for means" analysis indicated that the expression levels of the genes Os01g28690 and Os05g32120 were also closely correlated with the TN variation.

Os01g28690 encodes a nucleoporin autopeptidase domain-containing protein located in the center of LATN-3. We selected seven low TN varieties and 13 high TN varieties, which were correlated with the genotypes at LATN-3. We identified 24 allelic variations in both the promoter and gene coding regions. Fifteen, six 
Table 1 The 23 LATNs and their rice genomic information

\begin{tabular}{|c|c|c|c|c|c|c|c|}
\hline LATN & Name_of_Top-SNPs & SNP_Types & Chr. & Positions & $p$-value & Annotation & Reference \\
\hline 1 & SNP-1.5635230. & $\mathrm{A} / \mathrm{G}$ & 1 & $5,636,231$ & $1.04 \mathrm{E}-05$ & Novel_1 & / \\
\hline 2 & SNP-1.7037988. & $\mathrm{C} / \mathrm{G}$ & 1 & $7,038,989$ & 2.95E-05 & CCP1 & (Yan et al., 2015) \\
\hline 3 & SNP-1.16070366. & $A / G$ & 1 & $16,071,393$ & 4.56E-05 & Novel_2 & / \\
\hline 4 & SNP-1.18536011. & $A / G$ & 1 & $18,537,058$ & 4.03E-06 & Novel_3 & / \\
\hline 5 & SNP-1.36879662. & T/C & 1 & $36,880,706$ & $1.68 \mathrm{E}-05$ & Novel_4 & / \\
\hline 6 & SNP-1.37003809. & $\mathrm{C} / \mathrm{G}$ & 1 & $37,004,853$ & 6.07E-07 & Novel_5 & / \\
\hline 7 & SNP-1.37664127. & $A / G$ & 1 & $37,665,171$ & 1.56E-09 & REL 1, MOC2, PLA2 & $\begin{array}{l}\text { (Chen et al., 2015),(Koumoto et al., } \\
\text { 2013),(Kawakatsu et al., 2006) }\end{array}$ \\
\hline 8 & SNP-3.1121412. & $\mathrm{T} / \mathrm{C}$ & 3 & $1,122,415$ & $6.93 \mathrm{E}-10$ & OsDCL1 & (Zhang et al., 2015) \\
\hline 9 & SNP-4.19405464. & $\mathrm{C} / \mathrm{G}$ & 4 & $19,577,430$ & 1.31E-05 & OSAFB2,LAX2 & (Xia et al., 2012), (Tabuchi et al., 2011) \\
\hline 10 & SNP-4.33497579. & $T / G$ & 4 & $33,682,697$ & $5.26 \mathrm{E}-05$ & MOC3, OsGS2 & (Lu et al., 2015b), (Cai \& Xiao, 2010) \\
\hline 11 & SNP-5.7728359. & $C / T$ & 5 & $7,728,419$ & 5.05E-07 & Novel_6 & / \\
\hline 12 & SNP-5.18643585. & $C / T$ & 5 & $18,706,103$ & $4.52 \mathrm{E}-10$ & Novel_7 & / \\
\hline 13 & SNP-7.12702296. & $A / G$ & 7 & $12,703,290$ & 4.29E-05 & Novel_8 & / \\
\hline 14 & SNP-7.16935997. & $A / T$ & 7 & $16,936,991$ & $3.16 \mathrm{E}-07$ & Novel_9 & / \\
\hline 15 & SNP-8.19480736. & $A / G$ & 8 & $19,483,450$ & $2.72 \mathrm{E}-05$ & PAY1 & (Zhao et al., 2015) \\
\hline 16 & SNP-8.22337472. & $A / G$ & 8 & $22,340,186$ & $1.53 \mathrm{E}-08$ & Novel_10 & / \\
\hline 17 & SNP-8.26426233. & $\mathrm{T} / \mathrm{C}$ & 8 & $26,428,948$ & 4.74E-06 & OsPIN5b & (Lu et al., 2015a) \\
\hline 18 & SNP-9.4181935. & T/C & 9 & $4,182,936$ & $8.20 \mathrm{E}-07$ & Novel_11 & / \\
\hline 19 & SNP-9.22712391. & $A / T$ & 9 & $22,712,873$ & 5.89E-05 & OsAHP2 & (Sun et al., 2014) \\
\hline 20 & SNP-10.504597. & $A / G$ & 10 & 505,622 & 1.13E-06 & Novel_12 & / \\
\hline 21 & SNP-11.8537869. & $\mathrm{A} / \mathrm{C}$ & 11 & $8,543,260$ & 1.60E-06 & Novel_13 & l \\
\hline 22 & SNP-11.21947025. & $\mathrm{A} / \mathrm{C}$ & 11 & $22,413,155$ & $3.33 \mathrm{E}-10$ & Novel_14 & / \\
\hline 23 & SNP-11.26432763. & $\mathrm{T} / \mathrm{C}$ & 11 & $26,904,377$ & $1.02 \mathrm{E}-05$ & Novel_15 & I \\
\hline
\end{tabular}

and three of the allelic variations were located in the putative promoter, coding and UTR regions (Fig. 3), respectively. Five variations, including one bp Indel and four SNPs, were 100\% associated with the high/low TNs. Among the five top associated variations, three were located in the putative promoter region (bases 2076, 1561 and 1475 before the initiation codon) and two were located in the putative UTR regions (positions 4677 and
4719 , representing bases 42 and 84 after the stop codon, respectively). It is most notable that five allelic variations were located in the seventh exon of Os01g28690, four of which cause synonymous mutations (Fig. 3). However, one mutation (TG/GT), located on 4339 after the 'ATG' initiation codon, is a missense mutation, leading to the substitution of aa from tryptophan (W) coding by 'TGG' to valine (V) coding by 'GTG'.

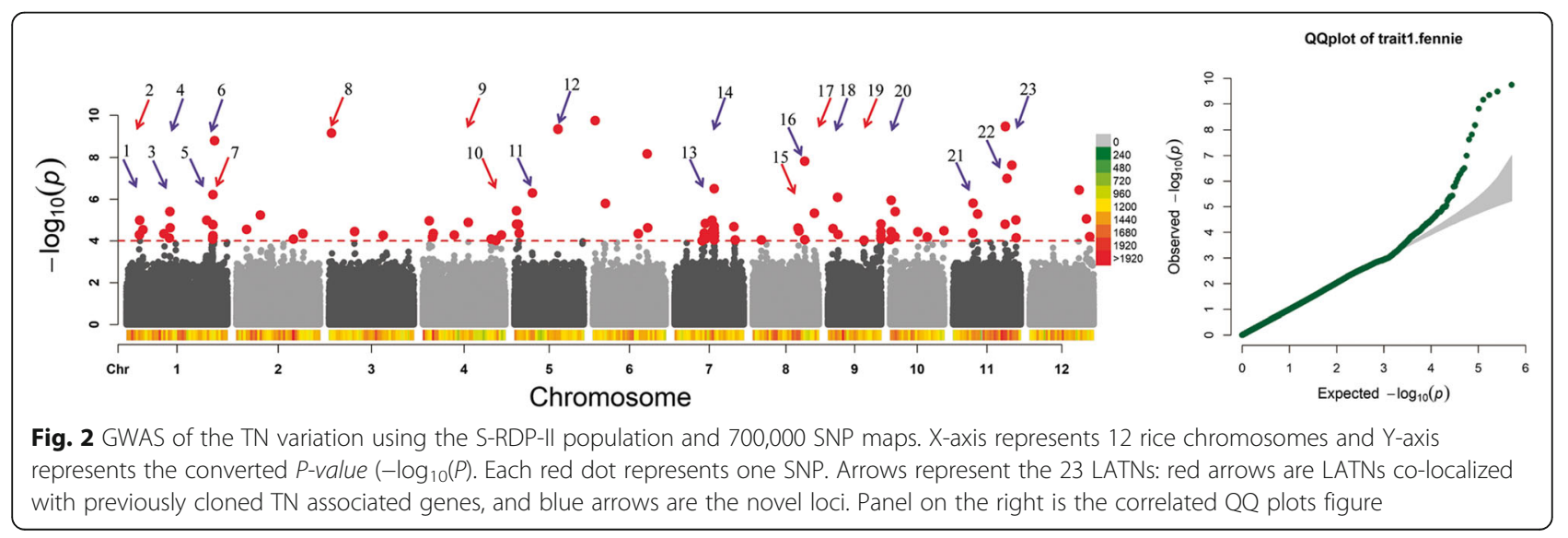




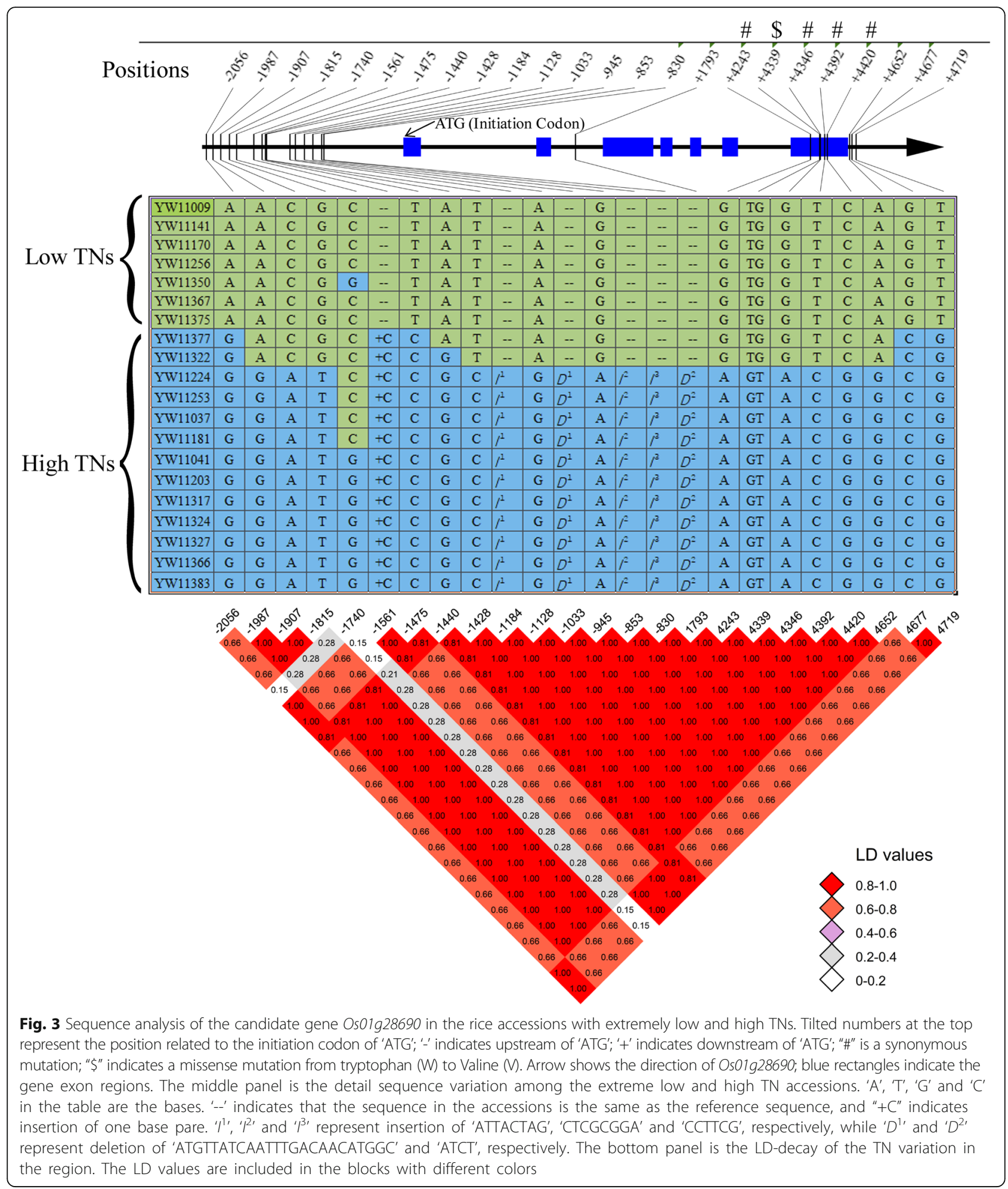

Os05g32120 encodes an alpha 1,3-xylosyltransferase, located in the center of LATN-12. Using six low TN varieties and seven high TN varieties, which are correlated with the genotypes at LATN-12, we identified 7 allelic variations from sequencing the full-length gene fragment
( $\sim 2 \mathrm{~kb}$ of the promoter and coding region, and $\sim 1 \mathrm{~kb}$ after the stop codon). Six of these variations were located in the putative promoter region (positions 1154, 1087, 1034, 1029, 911 and 832 before the initiation codon), and one was located in the second intron. Three 
allelic variations were $100 \%$ associated with the $\mathrm{TN}$ variation (Additional file 2: Figure S3).

Os07g28890 encodes an ethylene-responsive protein, which is located in the center of LATN-14. From the gene sequences of $17 \mathrm{TN}$ varieties (6 low and 11 high $\mathrm{TN}$ ), we identified seven allelic variations. Unlike other candidate genes, only three of the variations were located in the putative promoter region (positions 543, 472 and 408 before the initiation codon), and four variations were located within the gene. The first one was located in the first exon (G-to-A mutation) and caused a missense mutation from glycine $(\mathrm{G})$ to glutamic acid (E).

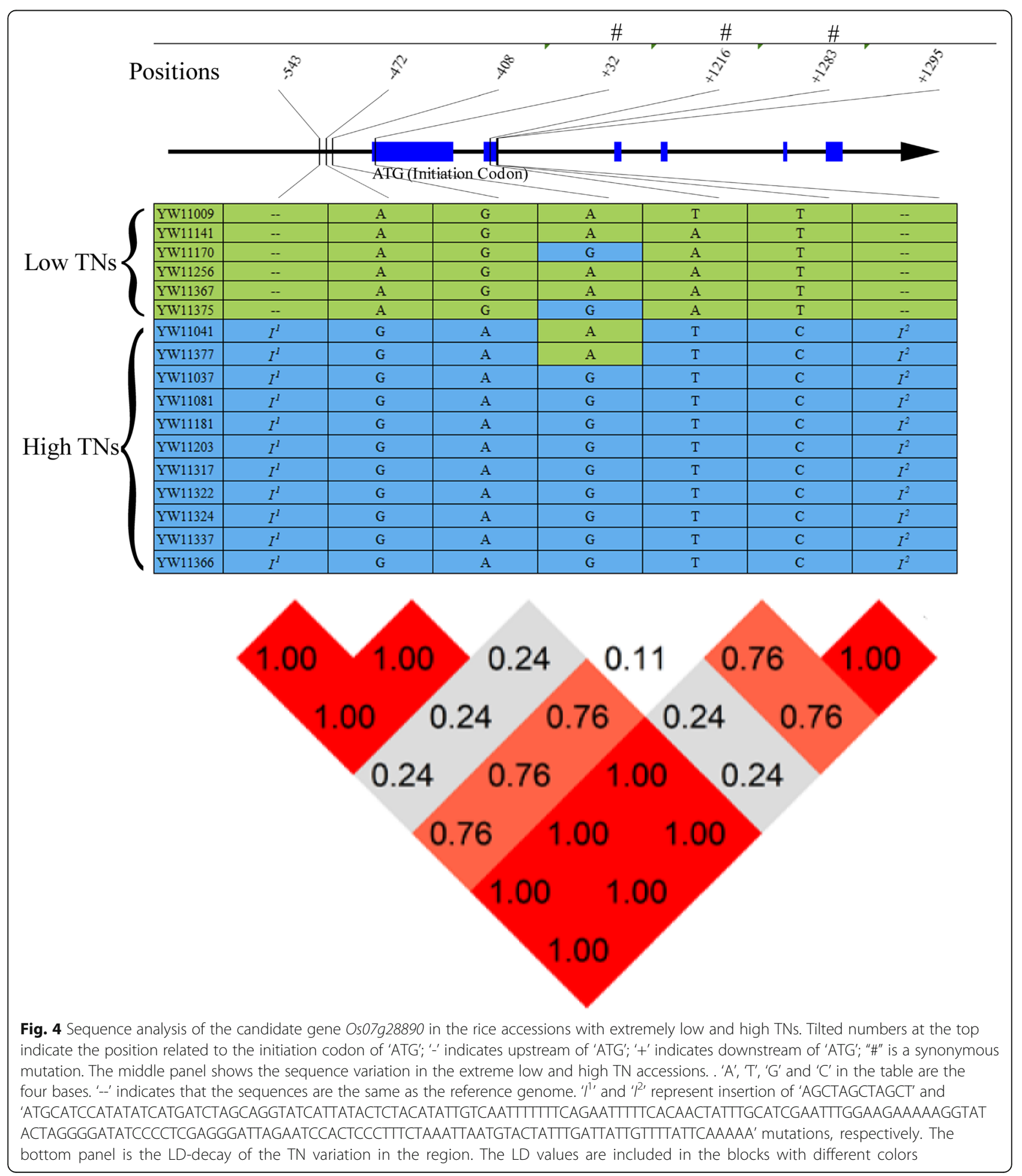


The second one was a synonymous mutation located in the second exon (A-to-T mutation) (Fig. 4). The remaining two were located in the second intron region: one very close to the GT--AG splicing site (GTAT...... AG, the fourth base ' $\mathrm{T}$ ' mutation to ' $\mathrm{C}$ '), and the other a $220 \mathrm{bp}$ deletion in the low TN varieties (or a $220 \mathrm{bp}$ insertion in the high TN varieties) close to the start of the splicing cite in the second intron (Fig. 4). We speculate that the mutations may lead to alternative splicing and functional changes in Os07g28890.

Os11g15130 and Os11g15210 are located in the middle of LATN-21. Os11g15130 encodes a jasmonate O-methyltransferase, and Os11g15210 encodes an anthocyanin regulatory protein. We analyzed the DNA sequences of 21 cultivars and identified that more than half (16 of 29) of the allelic variations of Os11g15130 were closely associated with TN variations. Three of the allelic variations were located in the last exon: one (base 2032 after the initiation codon, T/C SNP) lead from the proline (P) to serine (S) mutation; the second (base 2087 after the initiation codon region, T/C SNP) lead from the Alanine (A) to Valine (V) missense mutations; and the third (base 2096 after the initiation codon region, 'AATT' Indel) resulted in a frameshift mutation (Fig. 5). For Os11g15210, we identified very few allelic variations




(3 of 26) that were tightly associated with TN variations. Only one of these mutations was located in the exon region and was a synonymous mutation (Additional file 2: Figure S4).

As mentioned above, more than half of the allelic variations were closely associated with the $\mathrm{TN}$ variation and were located in the promoter/intron/UTR regions, rather than in the gene exon regions. We speculate that these variations may be associated with the expression levels of candidate genes. To test this speculation, we designed the gene specific primers (Additional file 1: Table S1) for qRT-PCR analysis of the five genes in the extreme low and high $\mathrm{TN}$ varieties. In addition, we performed the "two sample t-test for means" in the SAS system. The results indicated that the expression levels of two genes, Os01g28690 and Os05g32120, were positively (Os01g28690, $P=0.0132$ ) and negatively (Os05g32120, $P=$ 0.0098) associated with TN variations (Fig. 6).

In summary, we demonstrated that five genes were closely associated with TN variations in the S-RPD-II population, and the $\mathrm{TN}$ phenotypes are closely associated with the expression levels of two genes. Further functional analysis of the identified genes will help us better understand the genetic architecture of rice TN variations.

\section{Discussion}

Plant geneticists over a decade ago started to apply GWAS to dissect the genetic architecture of complex traits and map the loci that are associated with important agronomic traits (Feng et al. 2011). Numerous QTLs have been identified in more than 1000 GWAS projects that aimed to identify important loci in crops (Liu \& Yan 2019). In this study, we identified 23 LATNs using a subset of the RPD2 population and GWAS. Among them, 12 known TN-related genes are co-localized within the 8 LATNs regions, and the other 15 loci are novel. These results confirmed that the GWAS method is effective for identifying the TN loci in rice. To evaluate the GWAS results, we selected five candidate genes in the LATN regions and confirmed their associations with the TN variation at both the genomic DNA and

A

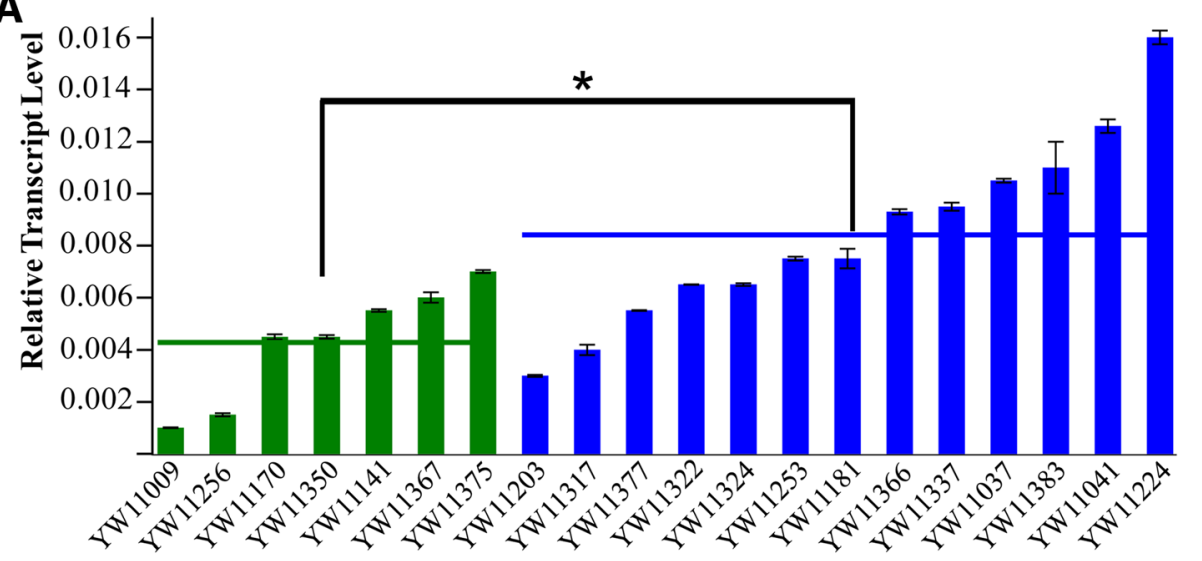

B

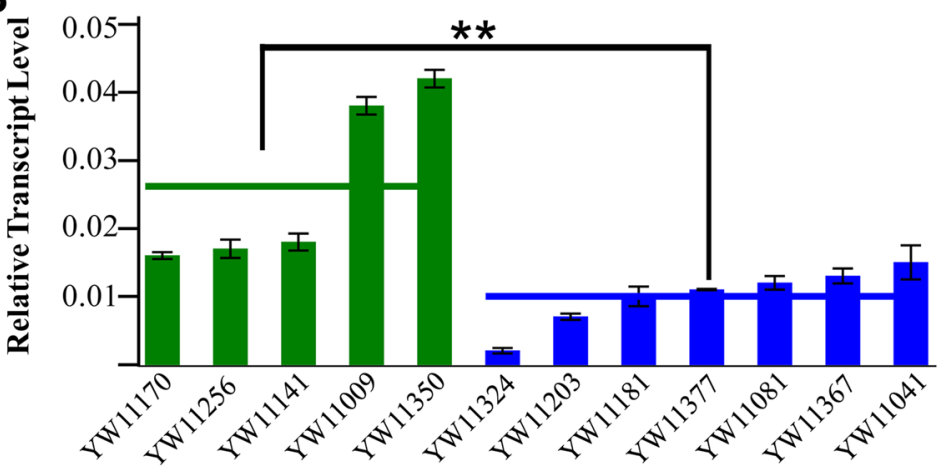

Fig. 6 qRT-PCR analysis of Os01g28690 (a) and Os05g32120 (b) between the extremely low and high TN accessions. X-axis represents the different rice accessions. Green bars are the low TN accession, and blue bars are the high TN accessions. Y-axis represents the relative gene transcript levels. The green and blue horizontal lines are the average gene expression levels in the low and high TN rice accessions, respectively. Black bars indicate the difference between the means of TNs in low and high TN accessions.'*' marks significant differences $(P=0.0132)$, while ${ }^{\prime * *^{\prime}}$ marks very significant differences $(P=0.0098)$ 
expression levels. Identification of these candidate genes has built a foundation for further detailed molecular analysis of their functions in rice tillering.

Previous studies showed that the genes involved in the phytohormone synthesis or degradation of strigolactone, auxin, cytokinin and carotenoid isomerase regulate or control the rice $\mathrm{TN}$ variation (Xu et al. 2005; Yeh et al. 2015; Liu et al. 2017). Ethylene is also known for regulating plant growth and development as well as regulating plant adaption to stressful conditions. Several important genes, such as ETO2 (Poupin et al. 2016), ERS1 (Deslauriers et al. 2015) and EIN2 (Guo et al. 2011), participate in regulating the ethylene signaling pathways in many plant growth processes. Additionally, jasmonate and its derivatives can regulate plant stress, growth and development (Wasternack 2007). In this study, we chose seven candidate genes, located either in the center of the peak value region or involved in the phytohormonal synthesis of the 15 novel LATNs, for further sequence and expression analyses. Two of the five candidate genes, Os07g28890 and Os11g15130, encode an ethyleneresponsive protein and a jasmonate O-methyltransferase protein, respectively. Knocking out and/or overexpressing of the two genes in transgenic rice will provide the information about their functions in rice tillering. Similar experiments can be performed for Os01g28690 and Os05g32120 because their expression levels are closely related to the $\mathrm{TN}$ variation.

\section{Conclusions}

In this study, we identified 23 LATNs using S-RDP-II and GWAS. Twelve known TN-related genes are colocalized within the eight LATNs regions, and 15 are novel LATNs. We demonstrated that five novel candidate genes are closely associated with $\mathrm{TN}$ variations at the DNA level. Interestingly, the promoter sequence variation and their expression levels in two of the five genes are closely associated with the TN variation, indicating that both gene and expression variations are important for the genetic architecture of the rice TN. The five identified novel candidate genes in this study are good genetic materials for dissecting the genetic architecture of rice tillering.

\section{Additional files}

Additional file 1: Table S1. Primers for PCR and $q R T-P C R$ analysis of the candidate genes. Table S2. TN phenotypes of S-RDP-II population in the field. Table S3. List of the detailed gene information of the 90 cloned TN variation associated genes. Table S4. The haplotype analysis of the Moc3 gene in the accessions with extreme low and high TN phenotypes. Table S5. The detailed information of the genes located in the 15 novel LATNs. Table S6. The seven candidate genes used for full length sequencing and expression analysis. (XLSX $50 \mathrm{~kb}$ )
Additional file 2: Figure S1. Field phenotypes of five low and five high TN accessions in Beijing, North of China. Figure S2. Field phenotypes of five low and five high TN accessions in Changsha, South of China. Figure S3. The haplotype analysis of the candidate gene Os05g32120 in the accessions with extremely low and high TN phenotypes. Figure S4. The haplotype analysis of the candidate gene Os11g15210 in the accessions with extremely low and high TN phenotypes. (DOCX 4965 kb)

\section{Acknowledgements}

We would like to thank Drs. Yuese Ning, Wende Liu and Xuli Wang for their help to the project.

\section{Authors' contributions}

HK and G-LW designed and initiated this project, provided the funding, and supervised the experiments. SJ, DW, SY, SL, BL and HK performed experiments. HK and G-LW analyzed the data. HK, SJ, and G-LW composed the manuscript. All authors have discussed the results and made comments on the manuscript. All authors have read and approved the final manuscript.

\section{Funding}

This research was supported by grants from the National Key R\&D Program of China (2017YFD0200900). This research was supported by grants from the International Cooperation Project of National Natural Science Foundation of China (31461143019), the National Natural Science Foundation of China to Houxiang Kang (31772120), and the Tianjin Science Foundation for Rice Molecular Marker System and Breeding Application (18YFZCNC91210).

\section{Availability of data and materials}

The datasets supporting the conclusions of this article are provided within the article and its additional files.

Ethics approval and consent to participate

No applicable.

\section{Consent for publication}

No applicable.

\section{Competing interests}

The authors declare that they have no competing interests.

\section{Author details}

${ }^{1}$ College of Agronomy, Hunan Agricultural University, Changsha 410128, China. ${ }^{2}$ State Key Laboratory for Biology of Plant Diseases and Insect Pest, Institute of Plant Protection, Chinese Academy of Agricultural Sciences, Beijing 100193, China. ${ }^{3}$ Tian Jin Key Laboratory of crop genetic breeding, Tianjin Crop Research Institute, Tianjin Academy of Agriculture Sciences, Tianjin 300112, China. ${ }^{4}$ Guangdong Key Laboratory of New Technology in Rice Breeding, Rice Research Institute, Guangdong Academy of Agricultural Sciences, Guangzhou 510640, China. ${ }^{5}$ Department of Plant Pathology, Ohio State University, Columbus, $\mathrm{OH}$ 43210, USA.

Received: 25 March 2019 Accepted: 27 May 2019

Published online: 20 June 2019

\section{References}

Alam MM, Tanaka T, Nakamura H, Ichikawa H, Kobayashi K, Yaeno T, Yamaoka N, Shimomoto K, Takayama K, Nishina H (2015) Overexpression of a rice heme activator protein gene (OsHAP2E) confers resistance to pathogens, salinity and drought, and increases photosynthesis and tiller number. Plant Biotechnol J 13:85-96Cai HM, Xiao JH (2010) Co-suppressed glutamine synthetase2 gene modifies nitrogen metabolism and plant growth in rice. Sci Bull 55:823-833

Chen QL, Xie QJ, Gao J, Wang WY, Sun B, Liu BH, Zhu HT, Peng HF, Zhao HB, Liu $\mathrm{CH}$ (2015) Characterization of rolled and erect leaf 1 in regulating leave morphology in rice. J Exp Bot 66:6047-6058

Deslauriers SD, Alvarez AA, Lacey RF, Binder BM, Larsen PB (2015) Dominant gainof-function mutations in transmembrane domain III of ERS1 and ETR1 suggest a novel role for this domain in regulating the magnitude of ethylene response in Arabidopsis. New Phytol 208:442-455 
Dong HJ, Zhao H, Xie WB, Han ZM, Li GW, Yao W, Bai XF, Hu Y, Guo ZL, Lu K, Yang $L$, Xing $Y Z$ (2016) A novel tiller angle gene, TAC3, together with TAC1 and D2 largely determine the natural variation of tiller angle in Rice cultivars. PLoS Genet 12:e1006412

Feng T, Bradbury PJ, Brown PJ, Hsiaoyi H, Qi S, Sherry FG, Rocheford TR, Mcmullen MD, Holland JB, Buckler ES (2011) Genome-wide association study of leaf architecture in the maize nested association mapping population. Nat Genet 43:159-162

Guo LQ, Ellis DE, Baggio-Saitovitch E (2011) EIN2 regulates salt stress response and interacts with a MA3 domain-containing protein ECIP1 in Arabidopsis. Plant Cell Environ 34:1678-1692

Huang XH, Wei XH, Sang T, Zhao Q, Feng Q, Zhao Y, Li CY, Zhu CR, Lu TT, Zhang ZW (2010) Genome-wide association studies of 14 agronomic traits in rice landraces. Nat Genet 42:961

Jiang L, Liu X, Xiong GS, Liu HH, Chen FL, Wang L, Meng XB, Liu GF, Yu H, Yuan YD, Yi W, Zhao LH, Ma HL, He YZ, Wu ZS, Melcher K, Qian Q, Xu HE, Wang YH, Li JY (2013) DWARF 53 acts as a repressor of strigolactone signalling in rice. Nature 504:401-405

Kang H, Wang Y, Peng S, Zhang Y, Xiao Y, Wang D, Qu S, Li Z, Yan S, Wang Z, Liu W, Ning Y, Korniliev P, Leung H, Mezey J, McCouch SR, Wang GL (2016) Dissection of the genetic architecture of rice resistance to the blast fungus Magnaporthe oryzae. Mol Plant Pathol 17:959-972

Kawakatsu T, Itoh J, Miyoshi K, Kurata N, Alvarez N, Veit B, Nagato Y (2006) PLASTOCHRON2 regulates leaf initiation and maturation in rice. Plant Cell 18: 612-625

Khush GS (2005) What it will take to feed 5.0 billion Rice consumers in 2030. Plant Mol Biol 59:1-6

Koumoto T, Shimada H, Kusano H, She K-C, Iwamoto M, Takano M (2013) Rice monoculm mutation moc2, which inhibits outgrowth of the second tillers, is ascribed to lack of a fructose-1,6-bisphosphatase. Plant Biotechnol 30:47-56

Li X, Qian Q, Fu Z, Wang Y, Xiong G, Zeng D, Wang X, Liu X, Teng S, Hiroshi F (2003) Control of tillering in rice. Nature 422:618

Lin H, Wang RX, Qian Q, Yan MX, Meng XB, Fu ZM, Yan CY, Jiang B, Su Z, Li JY (2009) DWARF27, an iron-containing protein required for the biosynthesis of strigolactones, regulates rice tiller bud outgrowth. Plant Cell 21:1512-1525

Liu HJ, Yan JB (2019) Crop genome-wide association study: a harvest of biological relevance. Plant J 97:8-18

Liu LH, Xie TT, Peng P, Qiu HY, Li XY (2017) Mutations in the MIT3 gene encoding a caroteniod isomerase lead to increased tiller number in rice. Plant Sci 267:1-10

Liu WZ, Chao W, Fu YP, Hu GC, Si HM, Li Z, Luan WJ, He ZQ, Sun ZX (2009) Identification and characterization of HTD2 : a novel gene negatively regulating tiller bud outgrowth in rice. Planta 230:649-658

Lu G, Coneva V, Casaretto JA, Ying S, Mahmood K, Liu F, Nambara E, Bi YM, Rothstein SJ (2015a) OsPIN5b modulates rice (Oryza sativa) plant architecture and yield by changing auxin homeostasis, transport and distribution. Plant J 83:913-925

Lu K, Wu BW, Wang J, Zhu W, Nie HP, Qian JJ, Huang WT, Fang ZM (2018) Blocking amino acid transporter OsAAP3 improves grain yield by promoting outgrowth buds and increasing tiller number in rice. Plant Biotechnol J 16: 1710-1722

Lu Q, Zhang MC, Niu XJ, Wang S, Xu Q, Feng Y, Wang CH, Deng HZ, Yuan XP, Yu HY (2015c) Genetic variation and association mapping for 12 agronomic traits in indica rice. BMC Genomics 16:1067

Lu ZF, Shao GN, Xiong JS, Jiao YQ, Wang J, Liu GF, Meng XB, Liang Y, Xiong GS, Wang YH (2015b) MONOCULM 3, an Ortholog of WUSCHEL in Rice, is required for tiller bud formation. J Genet Genomics 42:71-78

McCouch SR, Wright MH, Tung C-W, Maron LG, McNally KL, Fitzgerald M, Singh N, DeClerck G, Perez FA, Korniliev P, Greenberg AJ, Naredo MEB, Mercado SMQ, Harrington SE, Shi Y, Branchini DA, Kuser-Falcão PR, Leung H, Ebana K, Yano M, Eizenga G, McClung A, Mezey J (2016) Corrigendum: open access resources for genome-wide association mapping in rice. Nat Commun 7 : 11346

Poupin MJ, Greve M, Carmona V, Pinedo I (2016) A complex molecular interplay of auxin and ethylene signaling pathways is involved in Arabidopsis growth promotion by Burkholderia phytofirmans PSJN. Front Plant Sci 7:492-92.
Shinji I, Masahiko M, Tomotsugu A, Kazumitsu O, Itsuro T, Junko K (2005) Suppression of tiller bud activity in tillering dwarf mutants of rice. Plant Cell Physiol 46:79-86

Sun LJ, Zhang Q, Wu JX, Zhang LQ, Jiao XW, Zhang SW, Zhang ZG, Sun DY, Lu TG, Sun Y (2014) Two Rice authentic histidine Phosphotransfer proteins, OsAHP1 and OsAHP2, mediate Cytokinin signaling and stress responses in Rice. Plant Physiol 165:335-345

Tabuchi H, Zhang Y, Hattori S, Omae M, Shimizu-Sato S, Oikawa T, Qian Q, Nishimura M, Kitano H, Xie H, Fang XH, Yoshida H, Kyozuka J, Chen F, Sato Y (2011) LAX PANICLE2 of Rice encodes a novel nuclear protein and regulates the formation of axillary meristems. Plant Cell 23:3276-3287

Wang YH, Li JY (2005) The plant architecture of rice (Oryza sativa). Plant Mol Biol 59:75-84

Wang YH, Li JY (2011) Branching in rice. Curr Opin Plant Biol 14:94-99

Wasternack C (2007) Jasmonates: an update on biosynthesis, signal transduction and action in plant stress response, growth and development. Ann Bot 100: 681-697

Wu D, Guo ZL, Ye JL, Feng H, Liu JX, Chen GX, Zheng JS, Yan DM, Yang XQ, Xiong X (2019) Combining high-throughput micro-CT-RGB phenotyping and genome-wide association study to dissect the genetic architecture of tiller growth in rice. J Exp Bot 70:545-561

Xia KF, Wang R, Ou XJ, Fang ZM, Tian CG, Duan J, Wang YQ, Zhang MY (2012) OsTIR1 and OsAFB2 downregulation via OsmiR393 overexpression leads to more tillers, early flowering and less tolerance to salt and drought in Rice. PLOS One 7:364-373

Xu M, Zhu L, Shou HX, Wu P (2005) A PIN1 family gene, OsPIN1, involved in auxin-dependent adventitious root emergence and Tillering in Rice. Plant Cell Physiol 46:1674

Yan DW, Zhang XM, Zhang L, Ye SH, Zeng LJ, Liu JY, Li Q, He ZH (2015) Curved chimeric Palea 1 encoding an EMF1-like protein maintains epigenetic repression of OsMADS58 in rice Palea development. Plant J 82:12-24

Yasuno N, Yasui Y, Takamure I, Kato K (2007) Genetic interaction between 2 Tillering genes, reduced culm number 1 ( $r(n 1)$ and Tillering dwarf gene $d 3$, in Rice. J Hered 98:169

Yeh SY, Chen HW, Ng CY, Lin CY, Tseng TH, Li WH, Ku MSB (2015) Downregulation of Cytokinin oxidase 2 expression increases tiller number and improves Rice yield. Rice 8:36

Zhang DD, Liu MX, Tang MZ, Dong B, Wu DX, Zhang ZG, Zhou B (2015) Repression of microRNA biogenesis by silencing of OSDCL1 activates the basal resistance to Magnaporthe oryzae in rice. Plant Sci 237:24-32

Zhao L, Tan LB, Zhu ZF, Xiao LT, Xie DX, Sun CQ (2015) PAY1 improves plant architecture and enhances grain yield in rice. Plant J 83:528-536

\section{Publisher's Note}

Springer Nature remains neutral with regard to jurisdictional claims in published maps and institutional affiliations.

\section{Submit your manuscript to a SpringerOpen ${ }^{\circ}$ journal and benefit from:}

- Convenient online submission

- Rigorous peer review

- Open access: articles freely available online

- High visibility within the field

- Retaining the copyright to your article

Submit your next manuscript at $>$ springeropen.com 